\title{
The Janus face of myofibroblasts in the remodeling heart
}

Citation for published version (APA):

Hermans, K. C. M., Daskalopoulos, E. P., \& Blankesteijn, M. (2016). The Janus face of myofibroblasts in the remodeling heart. Journal of Molecular and Cellular Cardiology, 91, 35-41.

https://doi.org/10.1016/j.yjmcc.2015.11.017

Document status and date:

Published: 01/02/2016

DOI:

10.1016/j.yjmcc.2015.11.017

Document Version:

Publisher's PDF, also known as Version of record

Document license:

Taverne

Please check the document version of this publication:

- A submitted manuscript is the version of the article upon submission and before peer-review. There can be important differences between the submitted version and the official published version of record.

People interested in the research are advised to contact the author for the final version of the publication, or visit the DOI to the publisher's website.

- The final author version and the galley proof are versions of the publication after peer review.

- The final published version features the final layout of the paper including the volume, issue and page numbers.

Link to publication

\footnotetext{
General rights rights.

- You may freely distribute the URL identifying the publication in the public portal. please follow below link for the End User Agreement:

www.umlib.nl/taverne-license

Take down policy

If you believe that this document breaches copyright please contact us at:

repository@maastrichtuniversity.nl

providing details and we will investigate your claim.
}

Copyright and moral rights for the publications made accessible in the public portal are retained by the authors and/or other copyright owners and it is a condition of accessing publications that users recognise and abide by the legal requirements associated with these

- Users may download and print one copy of any publication from the public portal for the purpose of private study or research.

- You may not further distribute the material or use it for any profit-making activity or commercial gain

If the publication is distributed under the terms of Article $25 \mathrm{fa}$ of the Dutch Copyright Act, indicated by the "Taverne" license above, 


\title{
The Janus face of myofibroblasts in the remodeling heart
}

\author{
Kevin C.M. Hermans ${ }^{\text {a }}$, Evangelos P. Daskalopoulos ${ }^{\text {b,c }}$, W. Matthijs Blankesteijn ${ }^{\text {a,* }}$ \\ a Dept. of Pharmacology and Toxicology, Cardiovascular Research Institute Maastricht, Maastricht University, The Netherlands \\ b Pôle de Recherche Cardiovasculaire (CARD) Institut de Recherche Expérimentale et Clinique (IREC), Université catholique de Louvain (UCL), Brussels, Belgium \\ c Cardiovascular Research (Care) Institute, Athens \&' Ioannina, Greece
}

\section{A R T I C L E I N F O}

\section{Article history:}

Received 15 September 2015

Received in revised form 12 November 2015

Accepted 14 November 2015

Available online 12 December 2015

\section{Keywords:}

cardiac fibrosis

myofibroblast

epithelial-to-mesenchymal transition

fibrocyte

mesenchymal stem cell

signaling

\begin{abstract}
A B S T R A C T
Cardiac fibrosis is a process that is associated with multiple forms of cardiac remodeling. There is an ongoing debate whether fibrosis is good or bad for cardiac function. On the one hand, deposition of extracellular matrix is indispensable for the wound healing in the injured heart; on the other hand, interstitial fibrosis can lead to stiffening of the ventricular wall and adverse remodeling. A common denominator of cardiac fibrosis is the appearance of myofibroblasts that possess smooth muscle-like contractile properties and can synthesize extracellular matrix. Traditionally, these cells were considered to merely derive from resident fibroblasts in the ventricular wall. However, recent insights suggest that myofibroblasts can originate from cell types as diverse as epicardial cells, resident mesenchymal stem cells and circulating fibrocytes. In this review, we will describe the origin(s) of the myofibroblasts in different forms of cardiac remodeling. We will also address the question whether specific mediators that are involved in the transdifferentiation of these myofibroblasts from their precursors can be identified. This would be of relevance in order to design specific interventions that would attenuate the adverse fibrotic deposition whilst preserving the favorable aspects of the fibrotic response.
\end{abstract}

(c) 2015 Elsevier Ltd. All rights reserved.

\section{Contents}

1. Introduction . . . . . . . . . . . . . . . . . . . . . . . . . . . . . . . . . . . . . . . . . . . . . . 35

2. Characteristics of fibroblasts and myofibroblasts . . . . . . . . . . . . . . . . . . . . . . . . . . . . . . 36

3. Embryonic origin of cardiac fibroblasts . . . . . . . . . . . . . . . . . . . . . . . . . . . . . . . . . . . . . . . . . . . . 36

4. Myofibroblasts in the remodeling heart: good or bad? . . . . . . . . . . . . . . . . . . . . . . . . . . . . . . . . . 36

4.1. Myofibroblasts in the infarct area: good! . . . . . . . . . . . . . . . . . . . . . . . . . . . . . . . . . . . 36

4.2. Myofibroblasts in the cardiac interstitium: bad! . . . . . . . . 37

4.3. Myofibroblasts in heart valves: good? . . . . . . . . . . . . . . . . . . . . . . . . . . . . . . . . . . . . . . 37

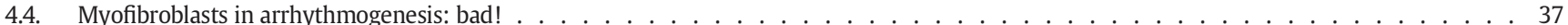

5. Origin of the myofibroblasts in the remodeling heart . . . . . . . . . . . . . . . . . . . . . . . . . . . . . . . . . . . . 37

5.1. The origin of myofibroblasts in the infarct area in permanent MI . . . . . . . . . . . . . . . . . . . . . . . . . . . . . . . . 38

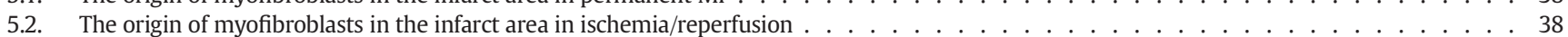

5.3. The origin of myofibroblasts in the interstitium . . . . . . . . . . . . . . . . . . . . . . . . . . . . . . . . . . 38

6. Perspective for selective intervention . . . . . . . . . . . . . . . . . . . . . . . . . . . . . . . . . . . . . 39

6.1. Fibrocyte attraction to the injured heart . . . . . . . . . . . . . . . . . . . . . . . . . . . . . . . . . . . . . . 39

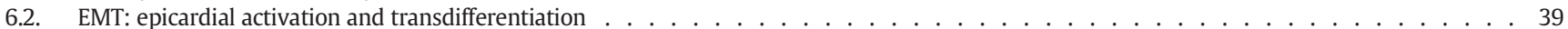

6.3. Mesenchymal stem cell transdifferentiation . . . . . . . . . . . . . . . . . . . . . . . . . . . . . . . . . . . . . . . . . 39

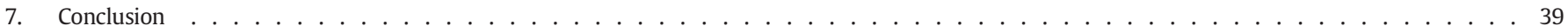

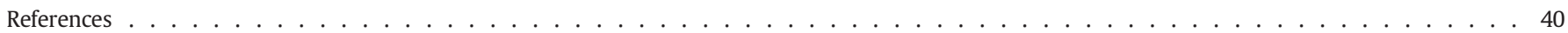

\footnotetext{
* Corresponding author.

E-mail address: wm.blankesteijn@maastrichtuniversity.nl (W.M. Blankesteijn).
}

\section{Introduction}

Cardiac remodeling is a term used to describe the alterations occurring in the myocardium due to a variety of stimuli. These stimuli can either be located within the heart (e.g. valvular dysfunction, loss of 
cardiomyocytes due to ischemia) or outside the heart (e.g. pressure overload due to aortic stenosis or increased peripheral vascular resistance). In either case, several common molecular mechanisms are activated in the remodeling heart. Examples of such mechanisms are inflammation and cardiac fibrosis. Several decades of research have taught us that these processes need to be tightly controlled in order to preserve an adequate cardiac function in the remodeling heart [1]. As an example, insufficient inflammation of the ischemic area after myocardial infarction (MI) leads to poor wound healing whereas an inflammatory response that is too strong can cause rupture of the infarct area $[2,3]$. Both will result in a deteriorated pump function of the heart and the latter condition will even cause instantaneous death when left untreated.

A similar picture can be drawn for cardiac fibrosis. In the normal, uninjured heart, a fixed amount of extracellular matrix (ECM) is present to preserve the architectural and biochemical integrity of the heart. This matrix is of vital importance to convey the forces generated by individual cardiomyocytes towards the atrial and ventricular cavities, allowing an effective circulation of the blood [4]. As this ECM is subject to wear and tear due to the mechanical load of the cardiac cycle, a certain amount of replacement (i.e. consisting of degradation of damaged fibers and synthesis of new matrix [5]) is required. To this end, the normal myocardium is populated with large numbers of cardiac fibroblasts (CF) which can outnumber the cardiomyocytes, depending on the species [6]. These cells are responsible for a balanced turnover of the ECM, leaving its total amount constant. This situation changes dramatically when the heart is exposed to excessive loading or when parts of the heart are injured. Under these conditions, differentiated fibroblasts called myofibroblasts (MFs), a cell type combining characteristics of fibroblasts and smooth muscle cells [7,8], appears in the heart. As previously noted for inflammation, this process can be beneficial under certain conditions, e.g. in the healing infarct, in order to prevent excessive dilatation of the left ventricle (LV). However, the presence of MF in the cardiac interstitium can also be detrimental for cardiac function as it can lead to excessive fibrosis, stiffening of the ventricular wall and disturbance of the electrical conductivity [9]. Therefore the cardiac MF can be seen as a cell type with two faces: indispensable for reparative processes but deleterious in adaptation to overload.

In this review, we will discuss cardiac fibrosis in the context of different pathological manifestations of cardiac remodeling, with specific focus on the ambiguous role of the MF. Furthermore, we will explore differences in MF origin and signaling that may be exploitable for selective therapeutic interventions to optimize the function of the remodeling heart.

\section{Characteristics of fibroblasts and myofibroblasts}

Fibroblasts are cells that are abundantly present in almost any organ or tissue of the body. In the uninjured heart, these cells are responsible for the synthesis of ECM, mainly consisting of collagen I and III $[6,10]$. Despite the fact that fibroblasts are indicated by a single name, they represent a rather heterogeneous population of mesenchymal cells [11]. Moreover, their development and transdifferentiation can be controlled by the local microenvironment, giving rise to a spectrum of different cellular phenotypes. At present, no specific molecular markers are known for fibroblasts, reflecting the diverse nature of these cells in different organs and tissues [9].

The versatility of the fibroblast is nicely illustrated by its ability to adapt to changing circumstances. In the heart, this is of particular relevance under conditions of stress such as pressure overload or ischemic injury. In a traditional view, the changes in mechanics and the stimulating factors that are released during these conditions evoke the transdifferentiation of fibroblasts into MF, a cell type characterized by stress fiber-like microfilament bundles containing $\alpha$-smooth muscle actin ( $\alpha$ SMA) [8]. The MF was originally described by Gabbiani et al. as a "modified fibroblast", derived from resident fibroblasts via transdifferentiation and playing a pivotal role in skin wound contraction [12]. In the meantime, more than four decades of research have tremendously increased our knowledge on MFs. Remarkably, MFs have been shown to be derived from multiple cell types, including endothelial cells, epithelial cells, pericytes, mesenchymal stem cells (MSC) and even from bone marrow-derived precursors, the so-called fibrocytes [13]. These observations dispute the traditional view that MFs can only be derived via transdifferentiation of resident CFs. This is supported by the observation that resident CFs, isolated from the heart, display limited proliferation in cell culture experiment $[14,15]$. It raises doubts whether resident CFs will be capable to undergo massive proliferation in the injured heart.

The transdifferentiation of MFs from their precursors is a complex sequence of events that has been investigated extensively. For a detailed description, we refer to review articles from our own group [16] as well as others [17]. Briefly, increased mechanical stress is a prerequisite for the formation of a proto-MF which is regarded to be the first step in MF transdifferentiation [8]. The second step is the interaction with stimuli such as transforming growth factor $\beta$ (TGF $\beta$ ) [18] and a splice variant of fibronectin called extra domain A (ED-A) [19]. However, the ongoing research in this area has offered a host of other MF-modulating factors, including Angiotensin II (Ang II), Endothelin I, Connective tissue Growth Factor (CTGF, now called CCN2) and Platelet-derived growth factor (PDGF) capable of either inducing or suppressing the MF phenotype [20]. As will be discussed in more detail below, these mediators appear to be uniformly required for MF transdifferentiation, irrespective of the nature or origin of the precursor cells.

\section{Embryonic origin of cardiac fibroblasts}

The origin of the resident CFs in the heart has been described in several excellent review articles [17,21]. Briefly, the main source of the interstitial CFs is the epicardium which is formed after the heart has looped. A sub-population of epithelial cells can undergo epithelial-tomesenchymal transition (EMT) and migrate into the walls of the developing heart to form a pool of resident CFs. These cells are generally referred to as epicardium-derived cells (EPDCs). EPDCs subsequently can differentiate into either coronary vascular smooth muscle cells or interstitial CFs [17]. The basic helix-loop-helix transcription factor TCF21 was found to be essential for this process as epicardial cells of TCF21-null mice were found to be unable to undergo EMT, resulting in hearts lacking fibroblasts [22]. Moreover, EPDC migration is essential for the proper formation of the heart, as it contributes to important structures such as the annulus fibrosis that forms the insulating layer between the atria and the ventricles, controlling transmission of electrical currents [23]. An additional source of CFs is the endothelium overlying the cardiac cushions, albeit that these fibroblasts mainly contribute to the heart valves [24].

\section{Myofibroblasts in the remodeling heart: good or bad?}

As mentioned above, the literature is quite divided about the role of the MF in the cardiac remodeling. To shed more light on this ambiguity, we will discuss the contribution of the MF to different processes that can take place in the remodeling heart in the following paragraphs.

\subsection{Myofibroblasts in the infarct area: good!}

Obstruction of the blood flow in a coronary artery results in ischemia of the parts of the heart that rely on this blood vessel for supply of oxygen and nutrients. Prolonged ischemia for more than a couple of hours leads to death of cardiomyocytes in the area. As adult cardiomyocytes only have minor regenerative capacity, loss of cardiomyocytes evokes a wound healing response. Cardiomyocyte death causes the release of pro-inflammatory stimuli which attracts polymorphonuclear neutrophils to the infarct. These cells commence clearing out the necrotic 
debris in the first days following MI. Next, monocytes invade the infarct area and differentiate into macrophages [25]. The cytokines and chemokines released by the inflammatory cells orchestrate the formation of granulation tissue in the injured areas. Granulation tissue typically contains newly-formed primitive blood vessels, macrophages and MF embedded in a primitive mesh of ECM. Eventually, the granulation tissue matures into a scar where the ECM becomes more crosslinked, the vasculature matures and MF numbers are reduced [10].

Having both contractile and synthetic properties, the MF appears to be well-equipped to optimize the wound healing process in the infarcted heart. First, the presence of microfilament bundles containing $\alpha S M A$ allows MFs to exert a sustained contractile force in the infarct area, thereby actively reducing its length. Particularly in the injured heart, where each contraction causes strain on the infarct area, this characteristic can be of value to prevent excessive dilatation of the infarct area. The MF can then stabilize the scar by deposition of newly synthesized ECM fibers, attenuating infarct expansion and preserving a favorable LV geometry [7].

Several lines of evidence illustrate that the persistent presence of MFs in the infarct area is beneficial for cardiac function. Well-healed human infarcts have been shown to contain MFs for many years after MI whereas dilated infarcts have lost their MFs prematurely [26]. Our lab as well as others have performed in vivo studies to further investigate this. In a study of infarct healing in mice of 5 different genetic backgrounds, a positive correlation was observed between MF numbers in the infarct area, reduction of LV dilatation and preservation of an adequate ejection fraction [27]. In the meantime, many interventions aimed at the MF in the infarct area have shown beneficial effects on cardiac function in animal models of MI. Briefly, the targets for these interventions can be as diverse as TGF $\beta$ signaling [18], Reactive oxygen species [28], Wnt signaling [29], microRNAs [30], and biomaterials releasing growth-promoting substances [31]. For a comprehensive overview of the therapeutic interventions aiming at wound healing process following MI, please refer to a recent review from our group [16]. The general picture emerging from all this work is that the prolonged presence of MFs in the infarct area is beneficial for maintenance of the ECM in the scar which is subject to wear due to the repetitive stretching. Strategies aiming at increasing the MF numbers in the infarct area offer an improved cardiac function and attenuate the development of heart failure post-MI [32]. However, we are not aware of studies in which these interventions were tested in MI patients.

\subsection{Myofibroblasts in the cardiac interstitium: bad!}

In contrast to ischemic heart disease, where the initial injury is localized to the ischemic areas of the heart, cardiac remodeling due to pressure overload evokes cardiac hypertrophy and fibrosis throughout the ventricles. Because this type of cardiac remodeling is typically observed in patients with hypertension, the name hypertensive heart disease (HHD) is frequently used to refer to this condition. Initially, this adaptation can normalize the wall stress, but in the long run it leads to diastolic dysfunction which frequently progresses towards combined diastolic/systolic dysfunction [33].

The pressure overload in HHD is typically associated with the appearance of MFs throughout the ventricular wall. This fibrosis is typically found in the endomysium, surrounding individual fibers of cardiomyocytes, and in the perimysium, surrounding bundles of cardiomyocyte fibers [34]. Moreover, excessive deposition of ECM can be found around the coronary arterial vasculature, a process referred to as perivascular fibrosis [35]. The excessive fibrosis not only increases the stiffness of the LV wall but can also affect the excitationcontraction coupling of the cardiomyocytes and the exchange of oxygen and nutrients. Therefore, the presence of MFs in the cardiac interstitium is generally considered to be detrimental for cardiac function [4].

\subsection{Myofibroblasts in heart valves: good?}

As indicated above, MFs are not observed in the normal healthy heart. There is, however, an exception to this observation as heart valves are known to contain MF under normal conditions. These MFs, also referred to as valvular interstitial cells (VICs), are involved in the continuous repair of damaged ECM caused by the stretch and deformation of the valve leaflets during the cardiac cycle [36]. Valvular disease can be accompanied by an activation of the MF, leading to the stiffening of the valve leaflets that is found in valvular stenosis [37]. These observations in the cardiac valves underscore the two faces of the MF: beneficial when appropriately controlled but detrimental when this control is lost.

\subsection{Myofibroblasts in arrhythmogenesis: bad!}

Cardiac fibrosis is known to increase arrhythmogenesis in various cardiovascular pathologies, as well as in the aged heart and the fibroblast/MF seem to play a key role [38]. The factors that mediate this phenomenon can be various: MF presence and ECM deposition in the myocardium themselves, gap junctions between MFs and myocytes, mechanical coupling of MFs to myocytes and paracrine factors released by the MFs have all been suggested as villains. Myocardial areas with "patchy" fibrosis or high interstitial fibrosis and areas with collagen presence in-between cardiomyocytes (diffuse fibrosis) possess very high potential for arrhythmogenesis, as they all lead to slow conductivity [39]. In vitro studies by Askar et al., demonstrated that high MF proliferation in cultures leads to arrhythmogenesis, however inhibition of MF proliferation is directly associated with a dramatic reduction of ectopic activity and attenuation of re-entrant arrhythmias [40]. Additionally, MFs appear to lose their arrhythmogenic properties when $\alpha S M A$ is disrupted [41]. The gap junction coupling MFs and myocytes is reported to be a source of arrhythmogenesis in both pressure overload [42] and ischemia [43]. The Askar group also showed that blunting Connexin 43 (Cx43) expression in the MF can have similar anti-arrhythmogenic effects, identifying $\mathrm{Cx} 43$ as a potential target for the attenuation of MF-mediated arrhythmogenesis [44]. Interestingly, the injection of autologous dermal fibroblasts into the infarct and peri-infarct areas of pigs following MI was reported to actually reduce ventricular tachycardias [45], while the group recently reported similar results in atrial fibrillation [46], disputing the theory of the "bad MF". It is suggested that the peri-infarct area is of crucial importance for the MF-mediated arrhythmogenesis. Low MF density in the peri-infarct area does not predispose to arrhythmia - as expected - however medium densities lead to action potential disturbances and consequently to arrhythmias. Surprisingly, high densities of MF appear to be antiarrhythmogenic, as they cause resting depolarization and thus reduce the chance for re-entrant arrhythmias [47]. To sum up, targeting MFs might be a rational strategy to prevent arrhythmogenesis in cardiac fibrosis, however further research is required in order to shed light into practical issues before this knowledge is translated to the clinic.

\section{Origin of the myofibroblasts in the remodeling heart}

The initial picture that arose from the work of Gabbiani et al. was that during wound healing the resident fibroblasts become activated and differentiate into MFs [12]. Although this concept was groundbreaking in its day, in the meantime new developments have showcased that MFs can arise from many different cell types [13]. Resident CFs are an unlikely source, as the proliferative capacity of these cells was found to be limited, at least in vitro [48]. In the following paragraphs, we will provide evidence that the MFs observed in different forms of cardiac remodeling can be derived from a variety of sources, which depend on the pathological entity that stimulates them. 


\subsection{The origin of myofibroblasts in the infarct area in permanent MI}

A careful analysis of the different stages of infarct healing suggests that it is quite unlikely that the MFs in the infarct area can be derived from fibroblasts that are locally present, as this area passes through a necrotic phase that is completely acellular [10]. This means that the MFs have to migrate from the border zone into the infarct area, a process that can easily be observed when infarct healing is followed in time in tissue sections stained for $\alpha$ SMA (Fig. 1). Although resident cardiac fibroblasts from the border zone have been shown to contribute to the pool of MF [14,15], these stainings also revealed a pool of $\alpha$ SMApositive cells at the epicardial and endocardial side of the heart. Interestingly, the appearance of $\alpha$ SMA-positive cells in the epicardial region appeared to precede the appearance of these cells in the infarct area as the pericardium at the site of infarction is restored at 3 days post-MI. Applying permanent ligation of the left ascending coronary artery in mice in which the Wilm's Tumor suppressor-1 (Wt1) lineage was genetically labeled, van Wijk et al. demonstrated that epicardium-derived mesenchymal cells contribute to the MF population in the infarct area [49], an observation that was recently confirmed [50]. The presence of MF from epicardial origin was also reported in a mouse model where $24 \mathrm{~h}$ of ischemia was followed by 7 days of reperfusion [51]; although technically speaking this is an ischemia/reperfusion study, the very long period of ischemia results in damage similar to permanent ischemia. Studies on zebrafish hearts have also shown that epicardialderived cells can differentiate into MF and confer cardio-protective effects following permanent myocardial damage due to cryoinjury [52]. These combined observations support the notion that epicardiumderived epithelial cells differentiate towards a mesenchymal phenotype via EMT, and are an important source of MFs in the infarct area after permanent MI.
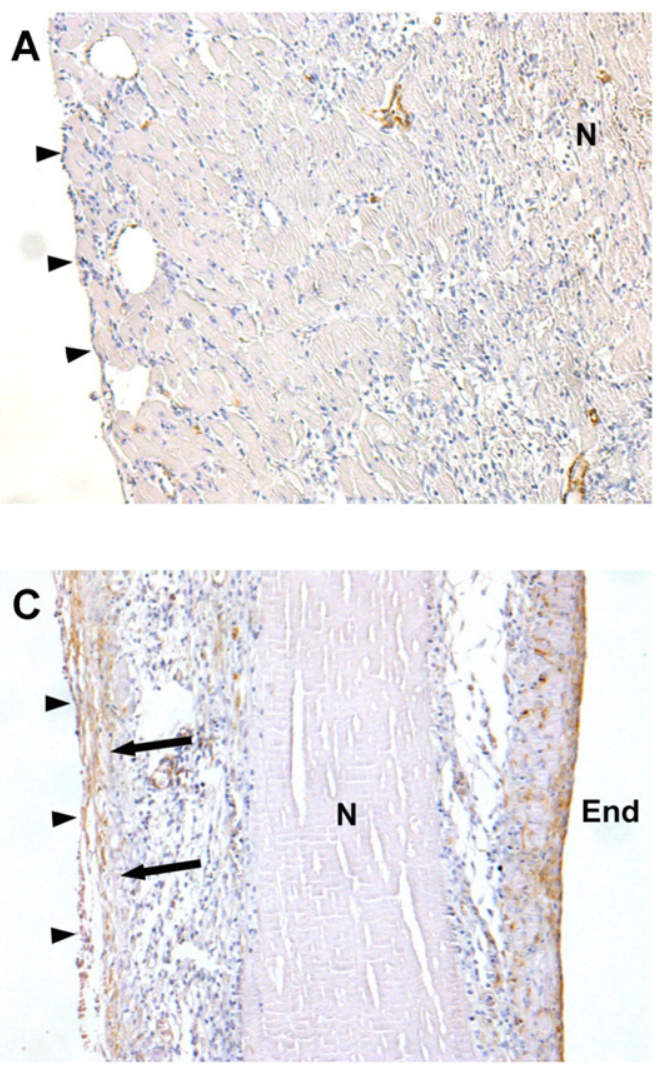

\subsection{The origin of myofibroblasts in the infarct area in ischemia/reperfusion}

In contrast to the permanent coronary artery ligation described above, in the ischemia/reperfusion model of MI the coronary artery is only occluded for a limited period of time (usually between 30 and $60 \mathrm{~min}$ ) and then re-opened to restore the blood flow to the injured area. Because of the fact that cardiomyocytes are much more susceptible to ischemia than most other cells that populate the ventricular wall [53], this procedure is likely to eliminate cardiomyocytes with relative selectivity. This may be illustrated by the study of Carlson et al., who observed fibroblast-like cells in the infarct area of the reperfused heart that deviate from structural fibroblasts by expressing CD44 and the primitive markers TERT, Nanog and CD34, all markers for MSC [54]. These fibroblast-like cells peaked at 3 days post-MI and transition towards MFs was observed in the following days [48]. In aged mice with reduced numbers of MSC, only few MSC-derived MFs were observed which significantly impaired the process of infarct healing [55]. From other studies, it was estimated that only a minority of the MF in the infarct area was bone marrow-derived [17]. This supports the conclusion that MSC are an important source of MFs in the injury caused by ischemia/reperfusion.

\subsection{The origin of myofibroblasts in the interstitium}

Several studies have addressed the origin of the MFs that populate the interstitium in models of pressure overload. The epicardium was excluded as a source of interstitial MFs in mice in which pressure overload was induced by either coarctation of the thoracic aorta or by infusion of Ang II [51]. In a model of daily 15 min periods of cardiac ischemia followed by reperfusion, interstitial fibrosis was observed that was mediated by fibroblasts originating from circulating precursors called
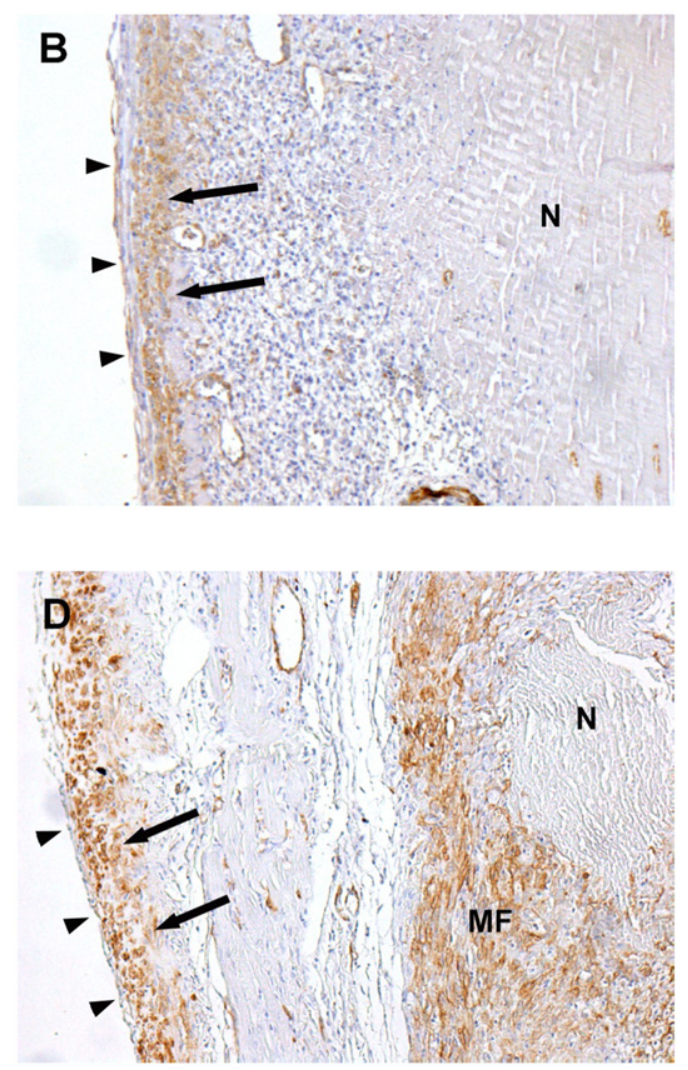

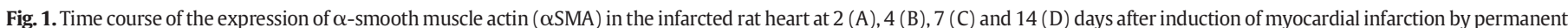

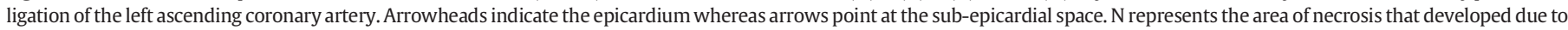

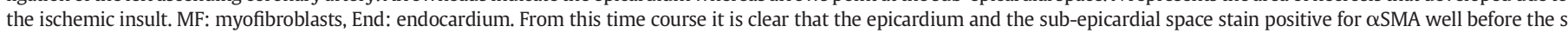
evolve around the necrotic area at day 14. Please note that the epicardial cells have become $\alpha$ SMA-negative at 14 days post-infarction. 
fibrocytes. The periods of ischemia in this model are too short to induce cardiomyocyte necrosis, but the procedure does result in the production of monocyte chemoattractant protein-1 (MCP-1) which is likely to be involved in attracting the fibrocytes. In mice lacking MCP1 the fibrotic response to the repetitive periods of ischemia was lost, underscoring the importance of this mechanism for the induction of fibrosis in this model.

Application of fate mapping and sex-mismatched bone marrow transplantations has shown that in a mouse model of pressure overload by coarctation of the aorta, one third of the fibroblasts and the majority of the MFs were derived from endothelial cells via EndMT (endothelialto-mesenchymal transition). Circulating bone marrow-derived fibrocytes accounted for less than $25 \%$ of the fibroblasts in this model. The EndMT was promoted by TGF $\beta$ and could be inhibited by administration of bone morphogenetic protein 7 [56]. In contrast, when pressure overload was induced by Ang II infusion, the bone marrow was the most prominent source of myocardial infiltrating MFs, whereas only few of these cells showed endothelial characteristics [57]. In a recent study from the Evans lab, however, both circulating fibrocytes and endothelial cells were disputed to be a source of fibroblasts in the pressure-overloaded heart. In contrast, in this study the fibroblasts present in the fibrotic heart were shown to originate from two resident fibroblast lineages: the endo- and epicardial-derived lineages [58]. The lack of well-defined and specific markers for (myo)fibroblasts is likely to have contributed to these conflicting data.

\section{Perspective for selective intervention}

MF transdifferentiation during cardiac remodeling has two faces: On the one hand, an adequate response to cardiac overload and injury would be impossible without fibrosis but, on the other hand excessive fibrosis - in particular of the interstitium - has detrimental effects on the pump function of the heart. Multiple factors, including TGF $\beta$, Endothelin-1, Ang II, CTGF/CCN2 and PDGF play important or even critical roles in MF transdifferentiation and the subsequent fibrotic response [20]. Unfortunately, these factors appear to have uniform roles in the different forms of fibrosis, making them unsuitable for selective intervention in the fibrotic response.

As illustrated above, there is a growing body of evidence that the MFs involved in various types of fibrotic response, originate from different sources. Since both the embryonic background and the routes towards differentiated MFs are likely to be diverse, this may open up opportunities for interventions targeting the adverse fibrotic response but leaving the beneficial fibrosis untouched. In the following paragraphs, we will discuss our current knowledge of the mediators involved in these different pathways.

\subsection{Fibrocyte attraction to the injured heart}

Fibrocytes are bone marrow-derived precursors of fibroblasts that are present in the circulation. These cell types express markers for bone marrow-derived cells such as CD34 and CD45, but are also positive for discoid domain receptor-2 (DDR2) and Collagen-I. In order to migrate to sites of injury, these cells have to be attracted by cytokines and/or chemokines. Therefore, these cells possess multiple chemokines including CCL2 (MCP-1), CCL5, CCL7 and express the receptors CCR2 and CXCR4 [59]. The attraction of fibrocytes from the circulation is a distinctive feature of these cells and therefore would qualify for a selective intervention. However, our knowledge of the chemokines responsible for fibrocyte attraction to the heart is rather limited. Until recently, the attraction of fibrocytes to the pressure-overloaded heart was considered to be CCL2- and CXCL12-dependent [60]. However, interrupting the signaling of CCR2 and CXCL4 via genetic and pharmacological intervention, respectively, did not reduce the invasion of fibroblasts in hearts of mice that were stimulated with Ang II for 3 days [61]. This illustrates that the gaps in our knowledge on the mediators attracting fibrocytes to the injured heart need to be filled before successful interventions can be performed.

Sun et al. showed that Cathepsin-L is a crucial regulator of fibrocyte mobilization and MF accumulation, as demonstrated by using Cathepsin-L knockout mice in a permanent ligation MI model [62]. An alternative way to restrict fibrosis by fibrocytes would be the prevention of their transdifferentiation. In mice exposed to daily short bouts of ischemia followed by reperfusion - a protocol that induces diffuse interstitial fibrosis - administration of serum amyloid $\mathrm{P}$ (member of the Pentraxin family) was shown to completely prevent cardiac fibrosis. This was accompanied by a marked reduction of $\mathrm{CD} 45^{+}$fibroblasts, known to be of myeloid origin [14]. This observation underscores that selective intervention in an adverse fibrotic mechanism may be feasible without interfering with beneficial fibrotic mechanisms.

\subsection{EMT: epicardial activation and transdifferentiation}

Epicardial cells respond to cardiac injury by reactivating a developmental gene expression program. It is therefore tempting to speculate that these activated epicardial cells can migrate into the myocardium and can - via EMT- differentiate into $\mathrm{CF}$ in a fashion similar to the population of the myocardium with EPDCs during development. Several members of the TGF $\beta$ superfamily have been shown to regulate EMT, but there are many other factors that can control EMT. These include Wt1, thymosin $\beta 4$, canonical Wnt signaling, PDGF and periostin [21]. In addition, Bronnum et al. showed recently that a complex crosstalking mechanism between the transcription factor Islet-1, microRNA-31 and $\beta$-catenin might be in place to regulate cardiac EMT [63]. The next step will be to study the effect of interventions in these mediators, either alone or in combination, on the contribution of EPDCs to the fibrosis in different models of cardiac remodeling.

\subsection{Mesenchymal stem cell transdifferentiation}

MSCs have been extensively studied in the context of cardiac regeneration by cell transplantation. A population of small precursor cells expressing primitive markers such as TERT, Nanog and CD34, but also fibroblast characteristics such as Collagen-I and DDR2, has been identified in the adult heart. At present, little is known about the growth factors and/or chemokines that induce the selective proliferation and migration of these cells and promote their transdifferentiation into (myo)fibroblasts. Cosentino et al. demonstrated that the GPR17 receptor is expressed in cells of mesenchymal origin and that it is co-localized with $\alpha S M A$ in the infarct area early in the wound healing process; this implyes that GPR17 could be playing a major role in the transdifferentiation of MF [64]. The potential role of pigment epithelium-derived factor (PEDF) in the transdifferentiation of MSCs to CF was reported by Liang et al., especially in aged MSCs [65]. Recent reports show that leptin [66], non-muscle myosin II (NMMII) [67] and adenosine A2B receptor [68] could also be implicated in MSC to MF transdifferentiaton. Lastly, one other mediator that has been suggested to trigger transdifferentiation of MSCs towards fibroblasts is insulin [69]. Elevated insulin levels tend to increase with age, which could serve as an explanation for the increased fibrosis in older hearts. However, as insulin does not appear to be a useful candidate for interventions in vivo, the underlying signaling pathway(s) will need to be further explored in order to develop this into a feasible intervention.

\section{Conclusion}

Cardiac fibrosis is a common process in the response of the heart to a plethora of stimuli. The appearance of MF is one of the common denominators in the fibrotic response. In some cases this response is required to preserve cardiac integrity and maintain an adequate function but in other cases it is detrimental and contributes to the development of dysfunction and heart failure. Recently, it has become clear that the classical 
view on cardiac fibrosis that MFs can only be derived from resident fibroblasts needs to be revised. In this review, we provide an overview of studies showing that MFs can be derived from various sources and that the contribution of these diverse progenitors to the pool of MF may vary in different forms of cardiac fibrosis. This knowledge may provide us with tools for selective intervention in the fibrotic response, targeting"bad" fibrosis but leaving the "good" fibrosis unaffected. However, there are several gaps in our knowledge that need to be filled before we can consider such an approach in the clinical setting. First, the vast majority of studies presented in this review are based on animal models of cardiac remodeling, mainly rodents. At present, we still have to find out how the concepts derived from these studies translate to humans. Complicating matters even further, different forms of fibrosis may go hand in hand in a single patient suffering from different cardiovascular disorders, e.g. hypertension and MI, as well as other comorbidities. Second, despite the different origin of the MF progenitors, many of the molecular mediators are common in the different forms of fibrosis. A better understanding of the molecular signaling pathways that induce the transdifferentiation of the MF phenotype from its different precursors will be essential to develop more precise interventions in the fibrotic response, allowing the attenuation of the noxious fibrosis while retaining or even stimulating the beneficial aspects of the fibrotic response.

\section{References}

[1] A. Papageorgiou, M. Rienks, Novel regulators of cardiac inflammation: matricellular proteins expand their repertoire, J. Mol. Cell. Cardiol. (2015) (in press).

[2] N.G. Frangogiannis, Regulation of the inflammatory response in cardiac repair, Circ. Res. 110 (2012) 159-173.

[3] F.A. van Nieuwenhoven, N.A. Turner, The role of cardiac fibroblasts in the transition from inflammation to fibrosis following myocardial infarction, Vasc. Pharmacol. 58 (2013) 182-188.

[4] P. Kong, P. Christia, N.G. Frangogiannis, The pathogenesis of cardiac fibrosis, Cell. Mol. Life Sci. 71 (2014) 549-574.

[5] M.L. Lindsey, R.P. Iyer, M. Jung, K.Y. DeLeon-Pennell, Y. Ma, Matrix metalloproteinases as input and output signals for post-Myocardial infarction remodeling, J Mol Cell Cardiol. (2015) (in press).

[6] P. Camelliti, T.K. Borg, P. Kohl, Structural and functional characterisation of cardiac fibroblasts, Cardiovasc. Res. 65 (2005) 40-51.

[7] S.W. van den Borne, J. Diez, W.M. Blankesteijn, J. Verjans, L. Hofstra, J. Narula, Myocardial remodeling after infarction: the role of myofibroblasts, Nat. Rev. Cardiol. 7 (2010) 30-37.

[8] B. Hinz, S.H. Phan, V.J. Thannickal, M. Prunotto, A. Desmouliere, J. Varga, et al., Recent developments in myofibroblast biology: paradigms for connective tissue remodeling, Am. J. Pathol. 180 (2012) 1340-1355.

[9] N.A. Turner, K.E. Porter, Function and fate of myofibroblasts after myocardial infarction, Fibrogenesis Tissue Repair 6 (2013) 5.

[10] J.P.M. Cleutjens, W.M. Blankesteijn, M.J.A.P. Daemen, J.F.M. Smits, The infarcted myocardium: simply dead tissue or a lively target for therapeutic interventions, Cardiovasc. Res. 44 (1999) 232-241.

[11] R.R. Driskell, B.M. Lichtenberger, E. Hoste, K. Kretzschmar, B.D. Simons, M. Charalambous, et al., Distinct fibroblast lineages determine dermal architecture in skin development and repair, Nature 504 (2013) 277-281.

[12] G. Gabbiani, G.B. Ryan, Presence of modified fibroblasts in granulation tissue and their possible role in wound contraction, Experientia 27 (1971) 549-550.

[13] E.M. Zeisberg, R. Kalluri, Origins of cardiac fibroblasts, Circ. Res. 107 (2010) 1304-1312.

[14] S.B. Haudek, Y. Xia, P. Huebener, J.M. Lee, S. Carlson, J.R. Crawford, et al., Bone marrow-derived fibroblast precursors mediate ischemic cardiomyopathy in mice, Proc. Natl. Acad. Sci. U. S. A. 103 (2006) 18284-18289.

[15] T. Yano, T. Miura, Y. Ikeda, E. Matsuda, K. Saito, T. Miki, et al., Intracardiac fibroblasts, but not bone marrow derived cells, are the origin of myofibroblasts in myocardial infarct repair, Cardiovasc. Pathol. 14 (2005) 241-246.

[16] E.P. Daskalopoulos, K.C. Hermans, W.M. Blankesteijn, Cardiac (myo)fibroblast: Novel strategies for its targeting following myocardial infarction, Curr. Pharm. Des. 20 (2014) 1987-2002.

[17] A. Deb, E. Ubil, Cardiac fibroblast in development and wound healing, J. Mol. Cell. Cardiol. 70 (2014) 47-55.

[18] M. Dobaczewski, W. Chen, N.G. Frangogiannis, Transforming growth factor (TGF)-beta signaling in cardiac remodeling, J. Mol. Cell. Cardiol. 51 (2011) 600-606.

[19] G. Serini, M.L. Bochaton-Piallat, P. Ropraz, A. Geinoz, L. Borsi, L. Zardi, et al., The fibronectin domain ED-A is crucial for myofibroblastic phenotype induction by transforming growth factor-beta1, J. Cell Biol. 142 (1998) 873-881.

[20] A. Leask, Getting to the heart of the matter: new insights into cardiac fibrosis, Circ. Res. 116 (2015) 1269-1276.
[21] J.C. Kovacic, N. Mercader, M. Torres, M. Boehm, V. Fuster, Epithelial-to-mesenchymal and endothelial-to-mesenchymal transition: from cardiovascular development to disease, Circulation 125 (2012) 1795-1808.

[22] A. Acharya, S.T. Baek, G. Huang, B. Eskiocak, S. Goetsch, C.Y. Sung, et al., The bHLH transcription factor Tcf21 is required for lineage-specific EMT of cardiac fibroblast progenitors, Development 139 (2012) 2139-2149.

[23] A.C. Gittenberger-de Groot, M.P. Vrancken Peeters, M.M. Mentink, R.G. Gourdie, R.E Poelmann, Epicardium-derived cells contribute a novel population to the myocardial wall and the atrioventricular cushions, Circ. Res. 82 (1998) 1043-1052.

[24] L.M. Eisenberg, R.R. Markwald, Molecular regulation of atrioventricular valvuloseptal morphogenesis, Circ. Res. 77 (1995) 1-6.

[25] N.A. Turner, Inflammatory and Fibrotic Responses of Cardiac Fibroblasts to Myocardial Damage Associated Molecular Patterns (DAMPs), J. Mol. Cell. Cardiol. (2016).

[26] I.E.M.G. Willems, M.G. Havenith, J.G.R. De Mey, M.J.A.P. Daemen, The $\alpha$-smooth muscle actin-positive cells in healing human myocardial scars, Am. J. Pathol. 145 (1994) 868-875.

[27] S.W. van den Borne, V.A. van de Schans, A.E. Strzelecka, H.T. Vervoort-Peters, P.M. Lijnen, J.P. Cleutjens, et al., Mouse strain determines the outcome of wound healing after myocardial infarction, Cardiovasc. Res. 84 (2009) 273-282.

[28] D. Sorescu, K.K. Griendling, Reactive oxygen species, mitochondria, and NAD(P)H oxidases in the development and progression of heart failure, Congest. Heart Fail. 8 (2002) 132-140.

[29] K.C. Hermans, W.M. Blankesteijn, Wnt Signaling in Cardiac Disease, Compr. Physiol. 5 (2015) 1183-11209.

[30] P.A. Da Costa Martins, L.J. De Windt, Targeting microRNA targets, Circ. Res. 111 (2012) 506-508.

[31] E.P. Daskalopoulos, A.D. Vilaeti, E. Barka, P. Mantzouratou, D. Kouroupis, M Kontonika, et al., Attenuation of post-infarction remodeling in rats by sustained myocardial growth hormone administration, Growth Factors 1-9 (2015).

[32] H. Laeremans, E.P. Daskalopoulos, B. Janssen, W.M. Blankesteijn, Blocking of Wnt/ Frizzled Signaling Stops the Dilatation of the Left Ventricle After Myocardial Infarction, Circulation 124 (2011).

[33] M.H. Drazner, The progression of hypertensive heart disease, Circulation 123 (2011) 327-334.

[34] B.C. Berk, K. Fujiwara, S. Lehoux, ECM remodeling in hypertensive heart disease, J. Clin. Investig. 117 (2007) 568-575.

[35] K.T. Weber, Y. Sun, S.K. Bhattacharya, R.A. Ahokas, I.C. Gerling, Myofibroblastmediated mechanisms of pathological remodelling of the heart, Nat. Rev. Cardiol. 10 (2013) 15-26.

[36] I. Vesely, Heart valve tissue engineering, Circ. Res. 97 (2005) 743-755.

[37] S. Jana, R.T. Tranquillo, A. Lerman, Cells for tissue engineering of cardiac valves, J Tissue Eng. Regen. Med. (2015).

[38] S.D. Stuart, N.M. DJ, M.L. Lindsey, C.M. Ripplinger, The crossroads of inflammation, fibrosis, and arrhythmia following myocardial infarction, J. Mol. Cell. Cardiol. (2015) (in press)

[39] T.P. Nguyen, Z. Qu, J.N. Weiss, Cardiac fibrosis and arrhythmogenesis: the road to repair is paved with perils, J. Mol. Cell. Cardiol. 70 (2014) 83-91.

[40] S.F. Askar, A.A. Ramkisoensing, M.J. Schalij, B.O. Bingen, J. Swildens, A. van der Laarse et al., Antiproliferative treatment of myofibroblasts prevents arrhythmias in vitro by limiting myofibroblast-induced depolarization, Cardiovasc. Res. 90 (2011) 295-304.

[41] C. Rosker, N. Salvarani, S. Schmutz, T. Grand, S. Rohr, Abolishing myofibroblast arrhythmogeneicity by pharmacological ablation of alpha-smooth muscle actin containing stress fibers, Circ. Res. 109 (2011) 1120-1131.

[42] S.Y. Shin, W.M. Jo, T.J. Min, B.K. Kim, D.H. Song, S.H. Hyeon, et al., Gap junction remodelling by chronic pressure overload is related to the increased susceptibility to atrial fibrillation in rat heart, Europace 17 (2015) 655-663.

[43] Y. Zhang, E.M. Kanter, K.A. Yamada, Remodeling of cardiac fibroblasts following myocardial infarction results in increased gap junction intercellular communication, Cardiovasc. Pathol. 19 (2010) e233-e240.

[44] S.F. Askar, B.O. Bingen, J. Swildens, D.L. Ypey, A. van der Laarse, D.E. Atsma, et al. Connexin43 silencing in myofibroblasts prevents arrhythmias in myocardial cultures: role of maximal diastolic potential, Cardiovasc. Res. 93 (2012) 434-444.

[45] F. Tondato, K. Robinson, J. Cui, T. Goodchild, N. Chronos, N.S. Peters, Effects on arrhythmogenesis and arrhythmic threshold of injection of autologous fibroblasts into myocardial infarcts in adult pigs, J. Cardiovasc. Transl. Res. 5 (2012) 337-344.

[46] F. Tondato, H. Zeng, T. Goodchild, F.S. Ng, N. Chronos, N.S. Peters, Autologous derma fibroblast injections slow atrioventricular conduction and ventricular rate in atrial fibrillation in swine, Circ. Arrhythm. Electrophysiol. 8 (2015) 439-446.

[47] K.S. McDowell, H.J. Arevalo, M.M. Maleckar, N.A. Trayanova, Susceptibility to arrhythmia in the infarcted heart depends on myofibroblast density, Biophys. J. 101 (2011) 1307-1315.

[48] S. Carlson, J. Trial, C. Soeller, M.L. Entman, Cardiac mesenchymal stem cells contribute to scar formation after myocardial infarction, Cardiovasc. Res. 91 (2011) 99-107.

[49] B. van Wijk, Q.D. Gunst, A.F. Moorman, M.J. van den Hoff, Cardiac regeneration from activated epicardium, PLoS ONE 7 (2012) e44692.

[50] A. Ruiz-Villalba, A.M. Simon, C. Pogontke, M.I. Castillo, G. Abizanda, B. Pelacho et al., Interacting resident epicardium-derived fibroblasts and recruited bone marrow cells form myocardial infarction scar, J. Am. Coll. Cardiol. 65 (2015) 2057-2066.

[51] C.M. Braitsch, O. Kanisicak, J.H. van Berlo, J.D. Molkentin, K.E. Yutzey, Differential expression of embryonic epicardial progenitor markers and localization of cardiac fibrosis in adult ischemic injury and hypertensive heart disease, J. Mol. Cell. Cardiol. 65 (2013) 108-119.

[52] J.M. Gonzalez-Rosa, M. Peralta, N. Mercader, Pan-epicardial lineage tracing reveals that epicardium derived cells give rise to myofibroblasts and perivascular cells during zebrafish heart regeneration, Dev. Biol. 370 (2012) 173-186. 
[53] W. Chen, N.G. Frangogiannis, Fibroblasts in post-infarction inflammation and cardiac repair, BBA 2013 (1833) 945-953.

[54] J. Trial, K.A. Cieslik, M. Entman, Mesenchymal Stem Cell-derived Inflammatory Fibroblasts Mediate Interstitial Fibrosis in the Aging Heart, J. Mol. Cell. Cardiol. (2015) (in press).

[55] K.A. Cieslik, J. Trial, M.L. Entman, Defective myofibroblast formation from mesenchymal stem cells in the aging murine heart rescue by activation of the AMPK pathway, Am. J. Pathol. 179 (2011) 1792-1806.

[56] E.M. Zeisberg, O. Tarnavski, M. Zeisberg, A.L. Dorfman, M.M. JR, E. Gustafsson, et al., Endothelial-to-mesenchymal transition contributes to cardiac fibrosis, Nat. Med. 13 (2007) 952-961.

[57] M.J. Sopel, N.L. Rosin, T.D. Lee, J.F. Legare, Myocardial fibrosis in response to Angiotensin II is preceded by the recruitment of mesenchymal progenitor cells, Lab. Investig. 91 (2011) 565-578.

58] T. Moore-Morris, N. Guimaraes-Camboa, I. Banerjee, A.C. Zambon, T. Kisseleva, A. Velayoudon, et al., Resident fibroblast lineages mediate pressure overload-induced cardiac fibrosis, J. Clin. Investig. 124 (2014) 2921-2934.

59] A. Mackinnon, S. Forbes, Bone marrow contributions to fibrosis, BBA 2013 (1832) 955-961.

[60] J.R. Crawford, S.B. Haudek, K.A. Cieslik, J. Trial, M.L. Entman, Origin of developmental precursors dictates the pathophysiologic role of cardiac fibroblasts, J. Cardiovasc. Transl. Res. 5 (2012) 749-759.

[61] A. Falkenham, M. Sopel, N. Rosin, T.D. Lee, T. Issekutz, J.F. Legare, Early fibroblast progenitor cell migration to the AngII-exposed myocardium is not CXCL12 or CCL2 dependent as previously thought, Am. J. Pathol. 183 (2013) 459-469.
[62] M. Sun, M. Chen, Y. Liu, M. Fukuoka, K. Zhou, G. Li, et al., Cathepsin-L contributes to cardiac repair and remodelling post-infarction, Cardiovasc. Res. 89 (2011) 374-383.

[63] H. Bronnum, D.C. Andersen, M. Schneider, A.Y. Nossent, S.B. Nielsen, S.P. Sheikh, Islet-1 is a dual regulator of fibrogenic epithelial-to-mesenchymal transition in epicardial mesothelial cells, Exp. Cell Res. 319 (2013) 424-435.

[64] S. Cosentino, L. Castiglioni, F. Colazzo, E. Nobili, E. Tremoli, P. Rosa, et al., Expression of dual nucleotides/cysteinyl-leukotrienes receptor GPR17 in early trafficking of cardiac stromal cells after myocardial infarction, J. Cell. Mol. Med. 18 (2014) 1785-1796.

[65] H. Liang, H. Hou, W. Yi, G. Yang, C. Gu, W.B. Lau, et al., Increased expression of pigment epithelium-derived factor in aged mesenchymal stem cells impairs their therapeutic efficacy for attenuating myocardial infarction injury, Eur. Heart J. 34 (2013) 1681-1690.

[66] P. Chen, R. Wu, W. Zhu, Z. Jiang, Y. Xu, H. Chen, et al., Hypoxia preconditioned mesenchymal stem cells prevent cardiac fibroblast activation and collagen production via leptin, PLoS ONE 9 (2014) e103587.

[67] M.A. Ngo, A. Muller, Y. Li, S. Neumann, G. Tian, I.M. Dixon, et al., Human mesenchymal stem cells express a myofibroblastic phenotype in vitro: comparison to human cardiac myofibroblasts, Mol. Cell. Biochem. 392 (2014) 187-204.

[68] S. Ryzhov, B.H. Sung, Q. Zhang, A. Weaver, R.J. Gumina, I. Biaggioni, et al., Role of adenosine A2B receptor signaling in contribution of cardiac mesenchymal stemlike cells to myocardial scar formation, Purinergic Signal 10 (2014) 477-486.

[69] K.A. Cieslik, J. Trial, S. Carlson, G.E. Taffet, M.L. Entman, Aberrant differentiation of fibroblast progenitors contributes to fibrosis in the aged murine heart: role of elevated circulating insulin levels, FASEB J. 27 (2013) 1761-1771. 